\title{
Cell polarity protein CRB3 is an independent favorable prognostic factor for clear cell renal cell carcinoma
}

\author{
XIAONA MAO $^{1 *}$, PINGPING LI $^{1 *}$, YU REN $^{2}$, JUAN LI $^{1}$, CAN ZHOU $^{2}$, JIN YANG $^{2}$ and PEIJUN LIU ${ }^{1}$ \\ ${ }^{1}$ Center for Translational Medicine and ${ }^{2}$ Department of Oncology, The First Affiliated Hospital, \\ Xi'an Jiaotong University, Xi'an, Shaanxi 710061, P.R. China
}

Received September 16, 2014; Accepted November 4, 2014

DOI: $10.3892 /$ ijo.2014.2763

\begin{abstract}
Epithelial cells possess apical-basal polarity and loss of epithelial cell polarity contributes to tumorigenesis and cancer progression. The Crumbs (CRB) polarity protein plays a crucial role in epithelial polarity maintenance, apical membrane formation, and tissue morphogenesis. Although evidence is increasing on involvement of deregulated polarity proteins in cancers, little is currently known about the roles of the CRB (Drosophila), especially the roles of CRB3, a homolog of the CRB, in clear cell renal cell carcinoma (ccRCC). Studies have shown that CRB3 may act as a tumor suppressor in non-human mammalian cells; the study here was aimed to examine the expression status of CRB3 in ccRCC and the relationships between CRB3 expression and clinicopathologic parameters of ccRCC patients. Our results showed that CRB3 was weakly expressed in ccRCC tissues, but strongly expressed in adjacent normal kidney tissues. Patients with loss of CRB3 expression showed a significantly shorter overall survival (OS) than patients with positive CRB3 expression. Our results suggested that CRB3 may be an independent favorable prognostic factor for patients with ccRCC. We also found that overexpression of CRB3 restrained invasion and migration of 786-O cells and loss of CRB3 expression promoted invasion and migration of human embryonic kidney 293T (HEK 293T) cells. This finding may explain why the negative CRB3 expression was associated
\end{abstract}

Correspondence to: Dr Peijun Liu, Center for Translational Medicine, The First Affiliated Hospital, Xi'an Jiaotong University, 277 Yanta West Road, Xi'an, Shaanxi 710061, P.R. China

E-mail: liupeijun@mail.xjtu.edu.cn

Dr Jin Yang, Department of Oncology, The First Affiliated Hospital, Xi'an Jiaotong University, 277 Yanta West Road, Xi'an, Shaanxi 710061, P.R. China

E-mail: yangjin@mail.xjtu.edu.cn

"Contributed equally

Key words: cell polarity, CRB3, clear cell renal cell carcinoma, prognosis, invasion, migration with poor prognosis in human ccRCC. Altogether, our data demonstrated that CRB3 may be used as a new independent favorable prognostic factor for human ccRCC.

\section{Introduction}

Cell polarity is defined as asymmetrical cell shapes and/or asymmetrical protein distributions and cell functions and contributes to normal tissue integrity and development (1). Polarity complexes, formed by polarity proteins, are required for the maintenance of cell polarity. Three apical-basal polarity complexes have been identified to date. The Crumbs (CRB) complex is formed by CRB (CRB1-3 in mammals), protein associated with Lin seven 1 (PALS1) and PALS1-associated tight junction protein (PATJ) (2-4). The partitioning defective (PAR) complex comprises PAR3 and 6, and atypical protein kinase $\mathrm{C}$ (aPKC). The $\mathrm{CRB}$ and PAR complexes are localized in the apical membrane domain and promote apical-membrane-domain identity $(5,6)$. Their function is antagonized by the basolaterally localized Scribble complex. The Scribble complex is composed of lethal giant larvae ( $\mathrm{Lgl})$, scribble (Scrib) and discs large (Dlg), which work together to promote basolateral membrane identity (7).

Most human cancers originate from epithelial cells, a cell type in which polarity proteins play crucial roles in maintaining epithelial structures (8). Disruption of cell polarity is a hallmark of cancer. Recent evidence indicates that the expression of a number of polarity proteins is altered in human cancers. A study has shown that polarity protein PAR6, a component of the PAR complex, is overexpressed in breast cancer-derived cell lines and advanced primary human breast cancers (9). Another component of the PAR complex, aPKC protein, is also found to be increased in ovarian cancers and non-small cell lung cancers $(10,11)$. However, loss of PAR3 can promote breast cancer metastasis $(12,13)$. PAR6 and aPKC may act as tumor promoters while PAR3 may serve as a tumor suppressor (14). Numerous studies have shown that components of the Scribble complex were correlated with cancers. $\mathrm{Lg12}$, a homolog of $\mathrm{Lgl}$, is downregulated in colorectal cancer and loss of Lgl2 may be involved in metastasis (15), which suggest that Lg12 may be a tumor suppressor. Dlg1, a homolog of Dlg, is also a tumor suppressor and loss of Dlg1 promotes the acquisition of invasiveness (16). Interestingly, the roles of the polarity protein Scrib in tumorigenesis seem to be 
complicated: in some cases it induces cell migration, whereas in others it is associated with inhibition of invasion and metastasis (17-20). Although evidence is increasing on involvement of deregulated polarity proteins in cancers, little is currently known about the roles of the CRB (Drosophila), especially the roles of CRB3, a homolog of the CRB.

CRB3 is widely expressed in epithelial tissues. Previous experiments have suggested that CRB may act as a tumor suppressor in Drosophila imaginal disc epithelium (21). Loss of CRB3 expression promotes mammalian epithelial tumor progression, while restoration of CRB3 expression restrains cell migration and metastasis (22). These data suggest that CRB may possess tumor suppressive potential not only in Drosophila but also in mammalian cells. However, no study has been performed on the role of deregulated CRB3 in progression toward malignancy in human tumors. Renal cell carcinoma (RCC) accounts for 3\% of all malignant tumors. Patients with RCC have a high risk of relapse and metastasis (23). Clear cell renal cell carcinoma (ccRCC), a main histologic type of RCC, accounts for a majority of kidney cancer-associated deaths $(24,25)$. The prognosis of the disease is difficult to predict due to lack of effective clinical prognostic factors. In this study, we aimed to investigate the role of CRB3 in human ccRCC. We found that CRB3 protein was weakly expressed in human ccRCC but strongly expressed in the adjacent normal kidney tissues. This is also the first study to investigate the relationships between the expression of CRB3 and the clinicopathological features of ccRCC patients. We found that negative CRB3 expression was correlated with shorter overall survival (OS) in ccRCC patients. Altogether, our results showed that $\mathrm{CRB} 3$ may be an independent favorable prognostic factor for patients with ccRCC.

\section{Materials and methods}

Cell culture. All cell lines were obtained from Shanghai Institute of Biochemistry and Cell Biology, Chinese Academy of Sciences (Shanghai, China). According to the American Type Culture Collection (ATCC) (Manassas, VA, USA), 786-O and 769-P were classified as ccRCC cell lines and ACHN was classified as the RCC cell line. OS-RC-2 was the RCC cell line and was maintained as previously described (26). The human embryonic kidney 293T (HEK 293T) cell line and Madin-Darby canine kidney (MDCK) cell line were maintained in Dulbecco's modified Eagle's medium (DMEM) supplemented with $10 \%$ fetal bovine serum (FBS) and $1 \%$ penicillin-streptomycin (all from HyClone, Logan, UT, USA). The ccRCC cell lines 786-O and 769-P and RCC cell line ACHN were cultured in RPMI-1640 (HyClone) supplemented with $10 \%$ FBS and $1 \%$ penicillin-streptomycin. The cells were incubated in $5 \% \mathrm{CO}_{2}$ at $37^{\circ} \mathrm{C}$.

Antibodies. The rabbit polyclonal anti-CRB3 antibody was purchased from Sigma-Aldrich which was used for immunohistochemistry (IHC) (1:200 dilution, HPA013835; St. Louis, MO, USA). The following antibodies were used for western blot analysis: rabbit polyclonal anti-CRB3 antibody (1:200 dilution, SC-292449; Santa Cruz Biotechnology, Inc., Santa Cruz, CA, USA), mouse monoclonal anti-E-cadherin antibody (1:500 dilution, ab1416; Abcam, Cambridge, MA, USA), mouse monoclonal anti-N-cadherin antibody (1:1,000 dilution, 610920; BD Biosciences, Bedford, MA, USA), rabbit polyclonal anti-vimentin antibody (1:2,000 dilution, 10366-1-AP) and mouse monoclonal anti-GAPDH antibody (1:5,000 dilution, HRP-60004) (both from Wuhan Sanying Biotechnology, Hubei, China). Anti-rabbit and anti-mouse HRP-conjugated secondary antibodies were purchased from Santa Cruz Biotechnology, Inc. (1:3,000 dilution, SC-2004 and SC-2005).

Transfection. The 786-O and HEK 293T cells were seeded in a 6-cm dish one day before transfection. Then the 786-O cells were transfected with GV168-CRB3 plasmid using TurboFect Transfection Reagent (Thermo Scientific, Rockford, IL, USA) to overexpress CRB3 according to the manufacturer's instructions. GV168-CRB3 plasmid was purchased from Genechem Co., Ltd. (Shanghai, China). The HEK 293T cells were transfected with siRNA using Lipofectamine 2000 (Life Technologies, Carlsbad, CA, USA) to knock down CRB3 expression according to the manufacturer's instructions. The siRNA was purchased from Shanghai GenePharma Co., Ltd. (Shanghai, China). Target sequences of oligonucleotides used were as follows: CRB3, 5'-UGG CAC UGU UGG UGC GGA ATT-3'; control (non-targeting), 5'-UUC UCC GAA CGU GUC ACG UTT-3'. The cells were harvested $48 \mathrm{~h}$ after transfection.

Tissue microarrays, patients and followup. The human study was approved by the Ethics Committee on Human Research of the First Affiliated Hospital of Xi'an Jiaotong University (Shaanxi, China). Written informed consent was obtained from all patients. Tissue microarrays (TMAs) were obtained from Shanghai Biochip Co., Ltd. (Shanghai, China). The TMAs contained 142 pairs of ccRCC and adjacent normal kidney tissues. Six pairs of tissue samples were excluded from the study due to lack of sufficient tumor tissue in the samples or fixation artifacts. The remaining 136 pairs of tissue samples consisted of tissues from 85 male and 51 female patients with a mean age of 59 years (range, 29-82 years). One-hundred and five patients were followed up for 2-74 months, with a median follow-up of 63 months. The follow-up data were summarized by the end of September, 2012. OS was defined as the period from the surgery to death or to the last known follow-up. Seventy-seven patients were still alive by the end of the follow-up.

Immunohistochemistry and scoring. The paraffin-embedded TMA slides were deparaffinized in xylene and rehydrated in ethanol with decreasing concentrations $(100,95,90$ and $85 \%$, $5 \mathrm{~min}$ each). After the slides were washed five times (3 min each time) with phosphate-buffered saline (PBS), $3 \% \mathrm{H}_{2} \mathrm{O}_{2}$ was used to block the endogenous peroxidase activity for $20 \mathrm{~min}$. After being rinsed, the slides were placed in $0.01 \mathrm{M}$ sodium citrate in a microwave oven $(\mathrm{pH} 6.0,100 \mathrm{~W}, 6 \mathrm{~min}$; $50 \mathrm{~W}, 13 \mathrm{~min}$ ) for antigen retrieval. Then slides were cooled at room temperature and added with goat serum to block non-specific binding sites for $15 \mathrm{~min}$. Without being washed, the slides were incubated with the primary antibody in a moist box at $4^{\circ} \mathrm{C}$ overnight. After being rinsed, the slides were incubated with the streptavidin-peroxidase staining system kit according to the manufacturer's instructions (SP-9001; Beijing 
Zhongshan Golden Bridge Biotechnology Co., Ltd., Beijing, China). Finally, the slides were stained by using the 3,3'-diaminobenzidine (DAB) liquid chromogen substrate kit (ZLI-9017; Beijing Zhongshan Golden Bridge Biotechnology Co., Ltd.) and counterstained with hematoxylin, followed by observation with Leica Microsystems GmbH (SCN 400; Mannheim, Germany). The results of IHC were evaluated individually in a blinded manner by three investigators who had no access to the clinical or clinicopathological status of the specimens. The intensity of immunohistochemical staining was scored as 0 (negative), 1 (weakly positive), 2 (moderately positive) or 3 (strongly positive). The extent of immunohistochemical staining was assessed according to the percentage of positive cells: 0 (negative), 1 (1-25\%), 2 (26-50\%), 3 (51-75\%) and $4(76-100 \%)$. The staining score for each field was calculated as the product of the intensity and extent of the staining. The final staining score for each sample was the mean of the staining scores of three randomly selected fields. The expression level was considered negative if the final score was $0-2$ and positive if the final score was 3-12. The expression level was considered low if the final score was 3-5 and high if the final score was 6-12.

CRB3 is not expressed in the adipose tissue according to the data provided in EBI Expression Atlas database (27). We performed IHC of the adipose tissue using the primary antibody rabbit polyclonal anti-CRB3 antibody and found that CRB3 protein was not expressed in the adipose tissue. CRB3 was expressed in the ccRCC tissues incubated with rabbit polyclonal anti-CRB3 antibody, and not expressed in the ccRCC tissues incubated with CRB3 recombinant protein and rabbit polyclonal anti-CRB3 antibody. Therefore, the ccRCC tissue incubated with CRB3 recombinant protein and primary antibody, and the adipose tissue were used as negative controls in the study. Tissues incubated with PBS in place of the primary antibody were also used as negative control to verify the specificity of the IHC results.

Real-time polymerase chain reaction analysis. Total RNA was extracted from cells by using the E.Z.N.A. ${ }^{\circledR}$ Total RNA kit I (Omega Bio-Tek, Inc., Norcross, GA, USA) according to the manufacturer's instructions. Reverse transcription was performed by using the PrimeScript ${ }^{\mathrm{TM}}$ RT Master Mix [Takara Biotechnology (Dalian) Co., Ltd., Liaoning, China] according to the manufacturer's instructions. Real-time polymerase chain reaction (real-time PCR) was performed at an annealing temperature of $60^{\circ} \mathrm{C}$ using the SYBR-Green I kit [Takara Biotechnology (Dalian) Co., Ltd.]. The following primers were used for real-time PCR reaction: CRB3 forward, 5'-CTT CTG CAA ATG AGA ATA GCA CTG T-3' and reverse, 5'-GAA GAC CAC GAT GAT AGC AGT GA-3'; GAPDH forward, 5'-CTC CTC CAC CTT TGA CGC TG-3' and reverse, 5'-TCC TCT TGT GCT CTT GCT GG-3'. Results were analyzed using the comparative $\mathrm{C}_{\mathrm{t}}$ method with the housekeeping gene glyceraldehyde-3-phosphate dehydrogenase (GAPDH) as an internal control.

Western blot analysis. Proteins were extracted from cells using RIPA lysis buffer (50 mM Tris- $\mathrm{HCl} \mathrm{pH} \mathrm{7.4,} 150 \mathrm{mM}$ $\mathrm{NaCl}, 1 \%$ Triton X-100, 0.1\% SDS, $1 \mathrm{mM}$ EDTA, and $1 \%$ sodium deoxycholate) supplemented with a protease inhibitor cocktail (Roche Diagnostics, Ltd., Basel, Switzerland). Cell lysates were centrifuged at $12,000 \mathrm{rpm}$ for $20 \mathrm{~min}$ at $4^{\circ} \mathrm{C}$. Proteins were separated by $12 \%$ SDS-polyacrylamide gel electrophoresis (SDS-PAGE) and transferred onto polyvinyl difluoride (PVDF) membranes $(0.22 \mu \mathrm{m}$; Millipore, Billerica, MA, USA) by electroblotting. The membranes were blocked with 5\% skimmed milk containing Tris-buffered saline and $0.1 \%$ Tween-20 (TBST) for $2 \mathrm{~h}$, followed by incubation with the primary antibody at $4^{\circ} \mathrm{C}$ overnight. After the membranes were washed with TBST, they were incubated with the secondary antibody. Immunoreactions were visualized using the chemiluminescence reagent (Millipore) according to the manufacturer's instructions. GAPDH was used as a protein loading control.

Wound-healing assay. 786-O and HEK 293T cells were transfected with GV168-CRB3 plasmid and siRNA, respectively. Cells were harvested $24 \mathrm{~h}$ after transfection and plated on 24-well cell culture plates. After $12 \mathrm{~h}$, scratch wounds were created manually in the monolayer of cells with a P200 pipette tip, washed twice with PBS, and incubated with fresh medium. The plates were photographed at the identical locations 0 and $24 \mathrm{~h}$ after incubation by Phase Contrast Microscope (20x; Nikon, Tokyo, Japan). Migration was determined by comparing the two sets of images.

Cell migration and invasion assays. Migration and invasion assays were performed in 24-well Transwells with polycarbonate filters ( $8 \mu \mathrm{m}$ pore size; Corning, Inc., Corning, NY, USA). The Transwell for the invasion assay was coated with Matrigel (1:5 dilution; BD Biosciences), while the Transwell for the migration assay was not coated. 786-O and HEK 293T cells were transfected with GV168-CRB3 plasmid and siRNA, respectively. The cells were harvested $24 \mathrm{~h}$ after transfection. 786-O cells were trypsinized and suspended with RPMI-1640 without FBS. 786-O cells, $2 \times 10^{4}$ (for migration assay) or $2 \times 10^{5}$ (for invasion assay), were added to the upper wells and $600 \mu 1$ RPMI-1640 medium containing 10\% FBS was added to the lower wells. Cells in the wells were incubated in 5\% $\mathrm{CO}_{2}$ at $37^{\circ} \mathrm{C}$ for $24 \mathrm{~h}$ for migration assay and $48 \mathrm{~h}$ for invasion assay. After incubation, cells in the upper wells were gently removed by scrubbing, fixed in $95 \%$ ethanol for $15 \mathrm{~min}$ and stained with $0.4 \%$ crystal violet for $30 \mathrm{~min}$. The number of transmembrane cells was calculated as the mean of the cell numbers in three randomly selected microscopic fields (20x; Nikon). Migration and invasion assays of HEK $293 \mathrm{~T}$ cells were performed following the same procedure except that HEK 293T cells were maintained with the DMEM medium and $5 \times 10^{4}$ cells were added for migration assay and $5 \times 10^{5}$ cells for invasion assay.

Statistical analysis. Statistical analyses were performed using the Statistical Package for the Social Sciences (SPSS) software (version 13.0; SPSS Inc., Chicago, IL, USA). The $\chi^{2}$ test was used to investigate the possible relationships between CRB3 expression and clinicopathological characteristics. The Mann-Whitney U test was used to compare differences between CRB3 protein expression in paired ccRCC and adjacent normal kidney tissues. Survival analysis was done by using the Kaplan-Meier curve and the log-rank test. Multivariate 


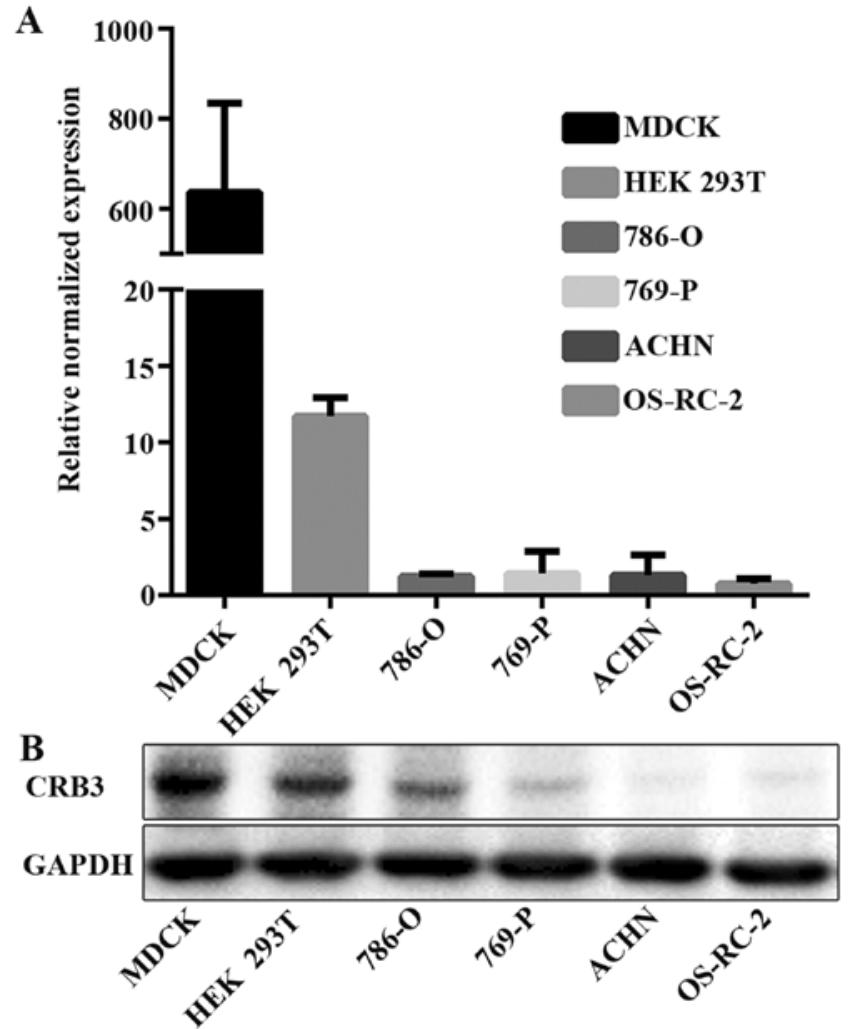

Figure 1. Crumbs (CRB)3 expression was low in clear cell renal cell carcinoma (ccRCC) and renal cell carcinoma (RCC) cell lines. (A) mRNA expressions of CRB3 in Madin-Darby canine kidney (MDCK), human embryonic kidney 293T (HEK 293T), ccRCC cell lines 786-O and 769-P, and RCC cell lines ACHN and OS-RC-2 were evaluated by real-time polymerase chain reaction (real-time PCR). Glyceraldehyde-3-phosphate dehydrogenase (GAPDH) was analyzed in parallel as a loading control. The values were expressed as mean \pm SEM. (B) Protein expressions of CRB3 in these cell lines were evaluated by western blot analysis. GAPDH was used as a protein loading control.

analysis was performed using the Cox proportional hazard regression model to explore the effects of CRB3 expression and ccRCC clinicopathological variables on survival. In cellular studies, values are expressed as mean \pm SEM. Comparisons between two groups were conducted using the Student's t-test. All experiments were done in triplicate. All statistical tests were two-sided. $\mathrm{P}<0.05$ was considered statistically significant.

\section{Results}

Low CRB3 expressions in ccRCC and RCC cell lines. We first examined CRB3 mRNA and protein expressions in the normal canine kidney epithelial cell line MDCK, HEK 293T cell line, ccRCC cell lines 786-O and 769-P, and the RCC cell lines ACHN and OS-RC-2. Real-time PCR and western blot analyses showed that MDCK had the highest mRNA and protein expression levels of CRB3 among the cell lines (Fig. 1A and B). The mRNA and protein expressions of CRB3 in ccRCC and RCC cell lines were relatively lower than those in MDCK.

Distributions of CRB3 protein in paired ccRCC and adjacent normal kidney tissues. Immunohistochemical analysis
Table I. Relationships between CRB3 expression and clinicopathologic parameters of ccRCC patients.

\begin{tabular}{|c|c|c|c|c|c|}
\hline \multirow[b]{2}{*}{ Variables } & \multirow[b]{2}{*}{ No. } & \multicolumn{2}{|c|}{ Expression levels } & \multirow[b]{2}{*}{$\chi^{2}$} & \multirow[b]{2}{*}{$\mathrm{P}$} \\
\hline & & Neg. & Pos. & & \\
\hline Age (years) & & & & 1.741 & 0.187 \\
\hline$<60$ & 76 & 49 & 27 & & \\
\hline$\geq 60$ & 60 & 45 & 15 & & \\
\hline Gender & & & & 0.230 & 0.632 \\
\hline Male & 85 & 60 & 25 & & \\
\hline Female & 51 & 34 & 17 & & \\
\hline Histologic grade & & & & 7.761 & $0.005^{\mathrm{b}}$ \\
\hline I-II & 98 & 61 & 37 & & \\
\hline III-IV & 38 & 33 & 5 & & \\
\hline TNM stage $^{c}$ & & & & 5.378 & $0.020^{\mathrm{a}}$ \\
\hline $1-2$ & 110 & 70 & 40 & & \\
\hline $3-4$ & 20 & 18 & 2 & & \\
\hline Tumor size $(\mathrm{cm})^{\mathrm{d}}$ & & & & 0.897 & 0.344 \\
\hline$<6.0$ & 73 & 53 & 20 & & \\
\hline$\geq 6.0$ & 63 & 41 & 22 & & \\
\hline Tumor site & & & & 0.431 & 0.512 \\
\hline Left & 72 & 48 & 24 & & \\
\hline Right & 64 & 46 & 18 & & \\
\hline Capsule invasion & & & & 0.197 & 0.657 \\
\hline Neg. & 113 & 79 & 34 & & \\
\hline Pos. & 23 & 15 & 8 & & \\
\hline
\end{tabular}

${ }^{\mathrm{a}} \mathrm{P}<0.05$; ${ }^{\mathrm{b}} \mathrm{P}<0.01$; ${ }^{\mathrm{c}} \mathrm{six}$ samples were excluded due to the absence of the TNM stage of these patients; ${ }^{\mathrm{d}}$ the patients were classified into two groups according to the mean value $(6.0 \mathrm{~cm})$ of their tumor sizes. CRB, Crumbs; ccRCC, clear cell renal cell carcinoma; Neg., negative; Pos., positive.

of TMAs was performed to investigate whether CRB3 was differently expressed in paired ccRCC and adjacent normal kidney tissues. The TMAs contained 136 pairs of ccRCC and adjacent normal kidney tissue samples. Due to the small sample size, low and high CRB3 expressions were classified as positive expressions. The clinicopathological features of the ccRCC patients and their CRB3 expression levels are listed in Table I. The specificity of the primary antibody rabbit polyclonal anti-CRB3 antibody was validated by using three negative controls (Fig. 2A). The results showed that CRB3 was not expressed in the adipose tissue and the ccRCC tissues incubated with CRB3 recombinant protein or PBS, which indicated that the primary antibody used for IHC was specific and the IHC results are sound. We found that CRB3 protein was predominantly expressed in the membrane and cytoplasm of renal tubular epithelial cells (Fig. 2B and C). CRB3 protein was strongly expressed in adjacent normal kidney tissues and weakly expressed in ccRCC tissues (Fig. 2B and C). Sixty-nine percent of ccRCC tissues showed complete loss of CRB3 expressions $(\mathrm{n}=136,69 \%, \mathrm{P}<0.001$, Table II). The expression level of CRB3 was significantly correlated with histologic grade 

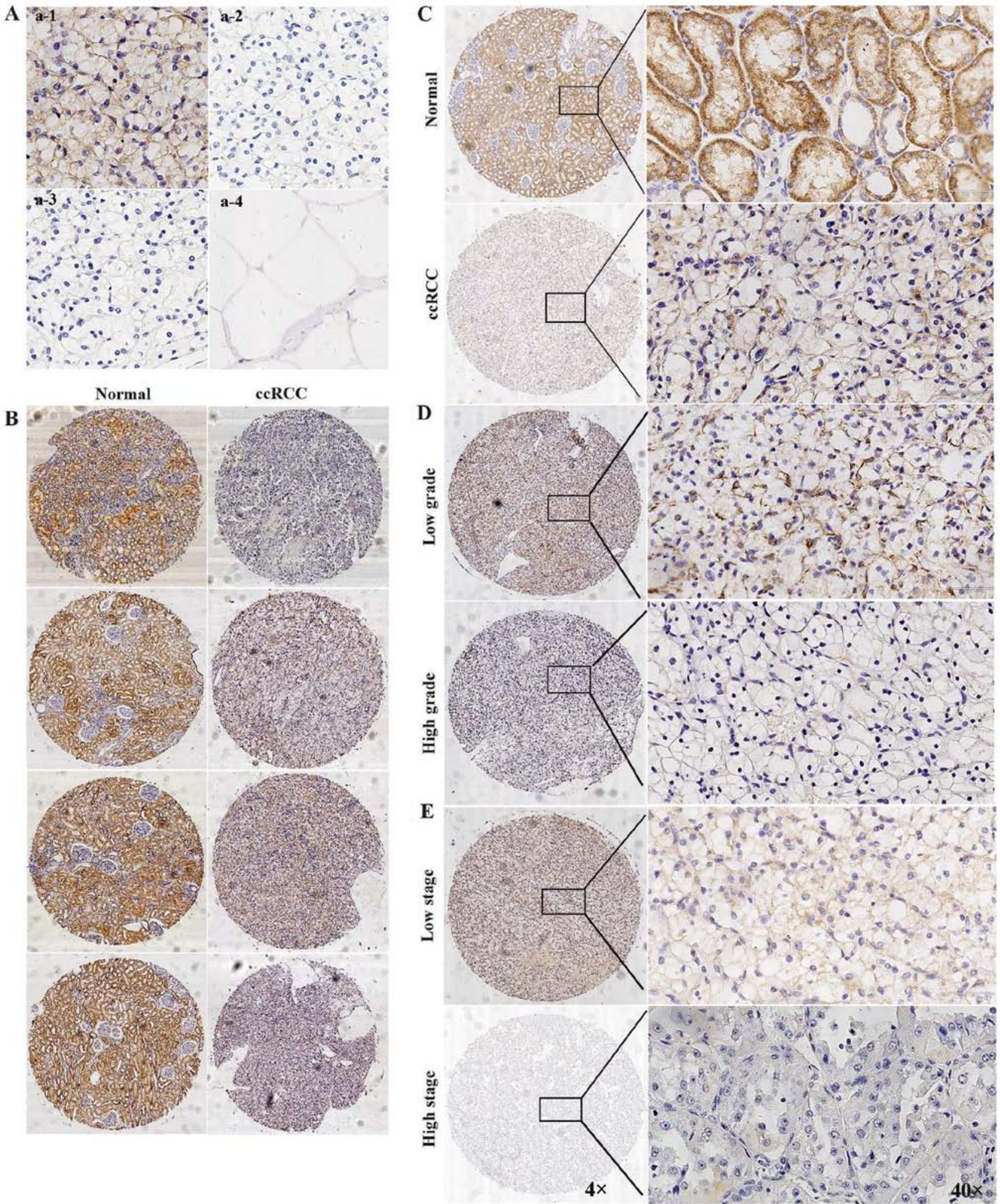

Figure 2. Crumbs (CRB)3 protein was weakly expressed in clear cell renal cell carcinoma (ccRCC) tissues. (A) CRB3 was expressed in the (a-1) ccRCC tissue, but not expressed in the (a-2) ccRCC tissue incubated with phosphate-buffered saline (PBS) in place of primary antibody or in the (a-3) ccRCC tissue incubated with CRB3 recombinant protein and primary antibody rabbit polyclonal anti-CRB3 antibody or in the (a-4) adipose tissue. The ccRCC tissue incubated with PBS in place of primary antibody, the ccRCC tissue incubated with CRB3 recombinant protein and primary antibody, and the adipose tissue were used as negative controls in the study. (B and C) The protein expression levels of CRB3 in paired ccRCC tissues and adjacent normal kidney tissues were evaluated by immunohistochemistry (IHC). Normal, adjacent normal kidney tissues. (D and E) The expression level of CRB3 was significantly correlated with histologic grade and TNM stage. Low histologic grade or TNM stage (histologic grade I-II or TNM stage 1-2); high histologic grade or TNM stage (histologic grade III-IV or TNM stage 3-4).

and TNM stage $(\mathrm{P}<0.05$, Fig. 2D and E, Table I), but not with age, gender, tumor size and site, and capsule invasion (Table I).
Prognostic significance of CRB3. The significant differences in the CRB3 expression between the pairs of ccRCC 

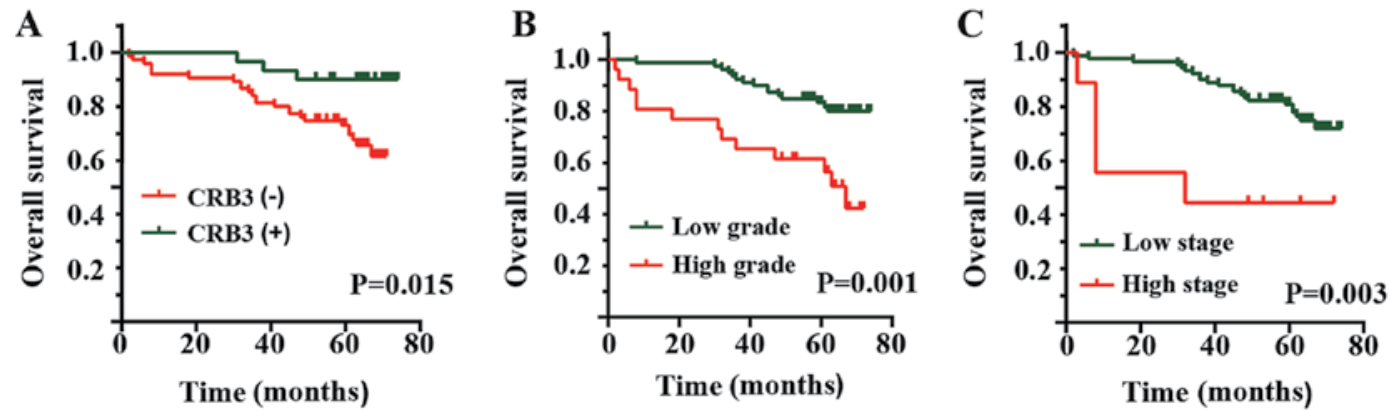

Figure 3. Kaplan-Meier curves of overall survival (OS) of clear cell renal cell carcinoma (ccRCC) patients. (A) Patients with negative Crumbs (CRB)3 expressions showed a significantly shorter OS than patients with positive CRB3 expression ( $\mathrm{P}=0.015$, log-rank test). (B and C) Patients with high histologic grade and TNM stage showed a significantly shorter OS than patients with low histologic grade and TNM stage (histologic grade I-II vs. III-IV, P=0.001; TNM stage $1-2$ vs. $3-4, P=0.003$, log-rank test).

Table II. CRB3 protein expressions in ccRCC and adjacent normal kidney tissues.

\begin{tabular}{|c|c|c|c|c|c|c|}
\hline \multirow[b]{2}{*}{ Variables } & \multirow[b]{2}{*}{ No. } & \multicolumn{3}{|c|}{ Expression levels } & \multirow[b]{2}{*}{4} & \multirow[b]{2}{*}{$\mathrm{P}$} \\
\hline & & Neg. & Low & High & & \\
\hline Normal $^{\mathrm{a}}$ & 136 & 11 & 48 & 77 & -10.175 & $<0.001$ \\
\hline ccRCC & 136 & 94 & 25 & 17 & & \\
\hline
\end{tabular}

aAdjacent normal kidney tissues. CRB, Crumbs; ccRCC, clear cell renal cell carcinoma; Neg., negative.

and adjacent normal kidney tissues inspired us to consider whether CRB3 could be used as a prognostic predictor for ccRCC patients. The association of CRB3 expression with OS was assessed by univariate analysis using the Kaplan-Meier curve and the log-rank test. No significant difference was detected in OS among patients with negative, low and high CRB3 expressions (data not shown). Due to the small sample size, low and high CRB3 expressions were classified as positive expressions. Of the 105 patients followed up for survival, 30 patients showed positive expression of CRB3 while the other 75 patients showed negative expression. Patients with negative CRB3 expressions had a significantly shorter OS than patients with positive expression $(\mathrm{P}=0.015$, log-rank test, Fig. 3A). The result indicated that CRB3 may be a prognostic indicator for patients with ccRCC. Univariate analysis also showed that patients with different histologic grades or TNM stages showed significantly different OS (data not shown). Here, due to the insufficient sample size, we divided the histologic grades or TNM stages into low histologic grade or TNM stage (histologic grade I-II or TNM stage 1-2) and high histologic grade or TNM stage (histologic grade III-IV or TNM stage 3-4). Log-rank test showed that high histologic grade or TNM stage was significantly associated with shorter OS (histologic grade I-II vs. III-IV, $\mathrm{P}=0.001$, Fig. 3B; TNM stage $1-2$ vs. $3-4, \mathrm{P}=0.003$, Fig. 3C).

Multivariate analysis was performed using the Cox proportional hazard regression model to further determine the effects of CRB3 expression and clinicopathological parameters on the prognosis of ccRCC patients. The multivariate Cox proportional hazard regression model mainly
Table III. Multivariate Cox proportional hazard regression analysis of overall survival in ccRCC patients. ${ }^{\mathrm{a}}$

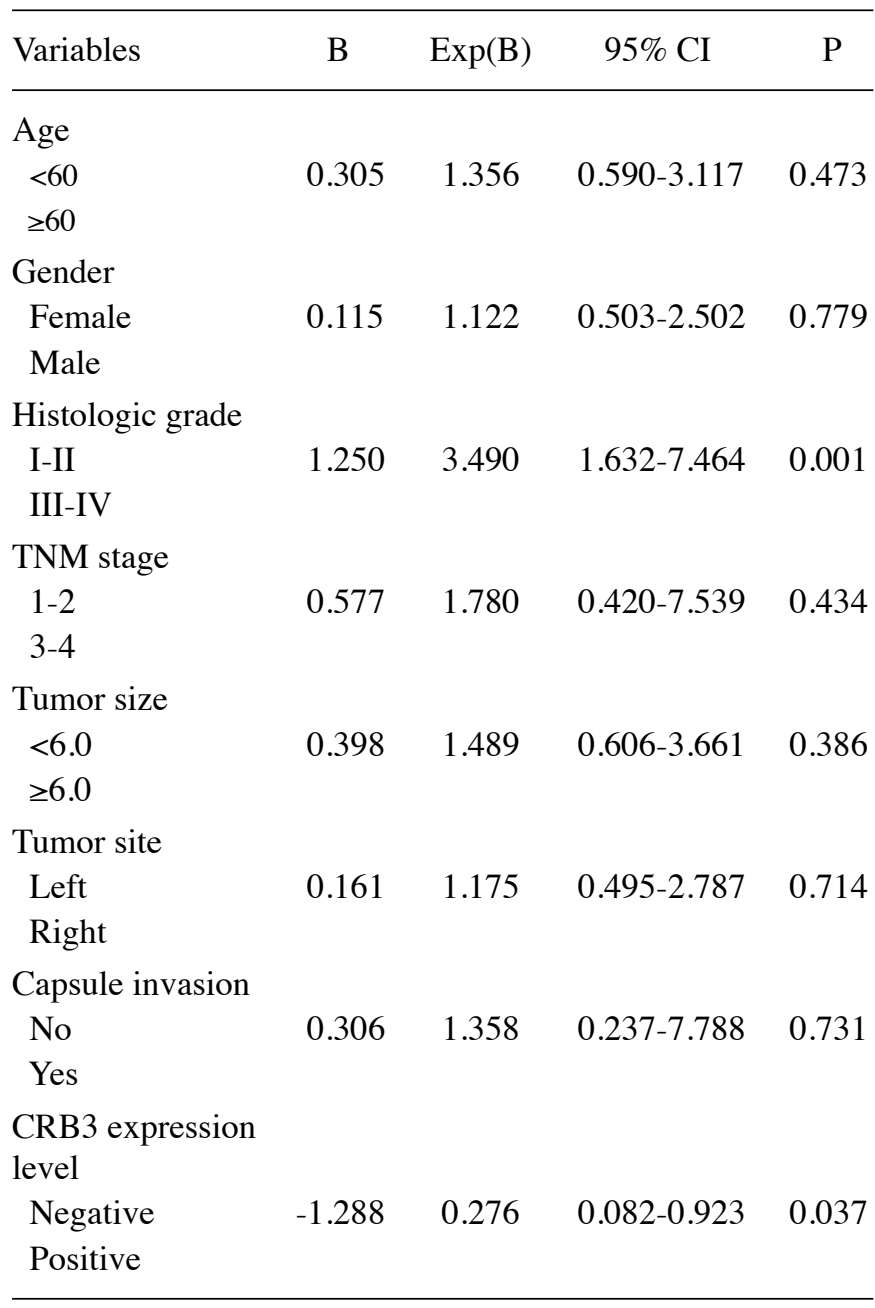

${ }^{\mathrm{a}} \mathrm{A}$ variable was entered into the model if its associated $\mathrm{P}$-value was $<0.05$. ccRCC, clear cell renal cell carcinoma.

considered CRB3 expression and clinicopathological parameters, including age, gender, histologic grade, TNM stage, tumor size and site, and capsule invasion. Results showed that positive $\mathrm{CRB} 3$ expression was linked to longer $\mathrm{OS}[\mathrm{B}=-1.288$, $\operatorname{Exp}(B)=0.276, P=0.037$, Table III], which indicated that 
positive CRB3 expression may be an independent favorable prognostic factor for OS and may be used to predict OS of ccRCC patients. Table III also shows that histologic grade may be a hazardous factor $[\mathrm{B}=1.250, \operatorname{Exp}(\mathrm{B})=3.490$, $\mathrm{P}=0.001$, Table III]. Age, gender, TNM stage, tumor size and site, and capsule invasion were not independent indicators for clinical outcome (Table III).

Roles of CRB3 in regulation of invasion and migration. Invasion and metastasis are the hallmarks of malignant tumor progression and the main causes of deaths in cancer. Here, cell migration, invasion and wound-healing assays were conducted to analyze the invasive and metastatic behavior in vitro. Since the 786-O cell line had a low protein expression of CRB3 and is a suitable host for transfection, 786-O cells were transfected with GV168-CRB3 plasmid to overexpress CRB3. Cell migration and invasion assays revealed that transfected 786-O cells showed reduced migration and invasion, when compared with control cells (Fig. 4A and B). Wound-healing assay showed that the wound of control cells disappeared, but the transfected cells failed to efficiently migrate to fill the void, indicating that the migration rate of 786-O cells was reduced after transfection with GV168-CRB3 plasmid (Fig. 4C). The results indicated that CRB3 overexpression significantly inhibited the migration and invasion of 786-O cells.

To investigate the effect of the loss of CRB3 on cell invasion and migration, HEK 293 T cells were transfected with siRNA to knock down the CRB3 expression. The transfected HEK 293T cells showed increased migration and invasion, when compared with control cells, indicating that loss of CRB3 expression may promote the migration and invasion of HEK 293T cells (Fig. 4D and E). Unfortunately, wound-healing assay was not successful for HEK 293T cells, probably due to the poor anchorage-dependent growth of the cells.

To investigate the roles of CRB3 in epithelial-mesenchymal transition (EMT), we examined the expression levels of the mesenchymal markers vimentin and $\mathrm{N}$-cadherin and the epithelial marker E-cadherin in 786-O cells transfected with GV168-CRB3 plasmid and HEK 293T cells transfected with siRNA. Western blot analysis revealed an increased E-cadherin expression and concomitant decreased vimentin and $\mathrm{N}$-cadherin expressions in transfected 786-O cells (Fig. 4F). However, transfection with siRNA induced decreased E- and N-cadherin expressions and an increased vimentin expression in HEK 293T cells (Fig. 4F). The expression changes of the mesenchymal and epithelial markers indicated that CRB3 may be associated with EMT.

\section{Discussion}

Loss of cell polarity has been widely assumed to be a key step in cancer progression. Most human cancers, including ccRCC, originate from epithelial cells, a cell type in which polarity proteins play crucial roles in maintaining epithelial structures (8). The CRB polarity complex, formed by CRB, PATJ and PALS1, participates in epithelial polarity maintenance, apical membrane formation, and tissue morphogenesis $(14,28)$. Previous studies have reported the association between disordered expressions of CRB and tumor progression in Drosophila and non-human mamma- lian cells. These reports inspired us to consider whether the deregulated CRB expression is associated with progression toward malignancy in human tumors, especially human ccRCC.

CRB3, one of the human CRB, is a small transmembrane protein with a conserved cytoplasmic domain $(29,30)$. We found that CRB3 was strongly expressed in normal kidney epithelial cells but weakly expressed in ccRCC and RCC cells. This led us to investigate the expression pattern of CRB3 in human tumor tissues. It has been found that CRB acts as a tumor suppressor in Drosophila imaginal disc epithelium (21). Loss of CRB3 expression promotes mammalian epithelial tumor progression while restoration of CRB3 expression restrains cell migration and metastasis (22). We found that ccRCC tissues had an apparently lower protein expression of CRB3 than adjacent normal kidney tissues, which is consistent with the above findings. CRB3 was predominantly expressed in the membrane and cytoplasm of renal tubular epithelial cells. CRB3 was detected in the cytoplasm probably because it is under dynamic intracellular trafficking control $(31,32)$ and in dynamic cycling and degradation processes. We also investigated the link between the expression of CRB3 and the prognosis of ccRCC patients, aiming to find an effective clinical prognostic marker for human ccRCC. Patients with negative CRB3 expression showed a significantly shorter OS than patients with positive expression. This result indicated that the positive CRB3 expression may be an independent favorable factor for OS. Due to the limited numbers of the tissue samples and histologic types, a study using a larger sample size is needed to verify these findings. 786-O cells transfected with GV168-CRB3 plasmid overexpressed CRB3 and showed remarkably lower migration and invasion, when compared with the control cells. HEK 293T cells transfected with siRNA had a decreased CRB3 expression and showed increased migration and invasion, when compared with control cells. The results indicated that loss of CRB3 expression may promote the cell migration and invasion. Since invasion and metastasis can lead to deaths of cancer patients, these findings may explain why ccRCC patients with negative CRB3 expressions had poor prognosis.

EMT is characterized by loss of cell polarity, enhanced cell motility and increased cell invasion (33). CRB3 expression is mediated by two EMT inducers, namely, ZEB1 and Snail, which suggests that CRB3 expression is associated with EMT $(15,34,35)$. We also found that CRB3 may be associated with EMT. Loss of CRB3 in HEK 293T cells decreased the E-cadherin expression and increased the vimentin expression. On the other hand, overexpression of $\mathrm{CRB} 3$ in 786-O cells increased the E-cadherin expression and decreased the vimentin expression. Dysfunctions of vimentin and E-cadherin have been considered hallmarks of EMT. Vimentin is involved in epithelial cell migration and invasion (36). Reduction in E-cadherin increases cell mobility and promotes tumor cell invasion; increase in E-cadherin has the reverse role (37-39). The changes in the expression of EMT markers may be the causes of altered cell migration and invasion. Thus, loss of CRB3 induced E-cadherin reduction and vimentin increase, which may lead to increase cell migration and invasion. Advanced cancers often undergo a partial or full EMT, which disrupts epithelial 
A

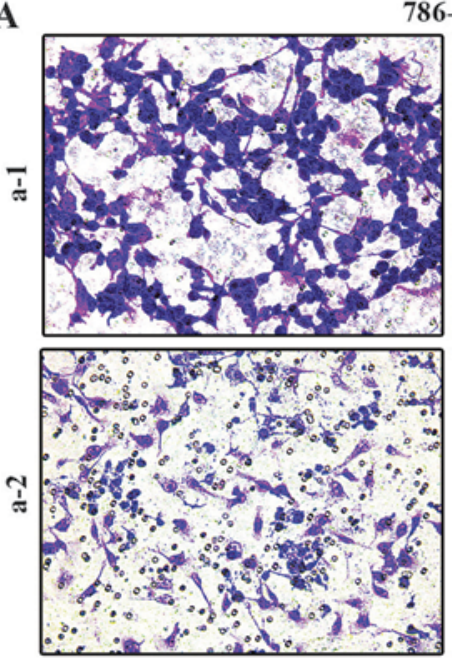

Control

C
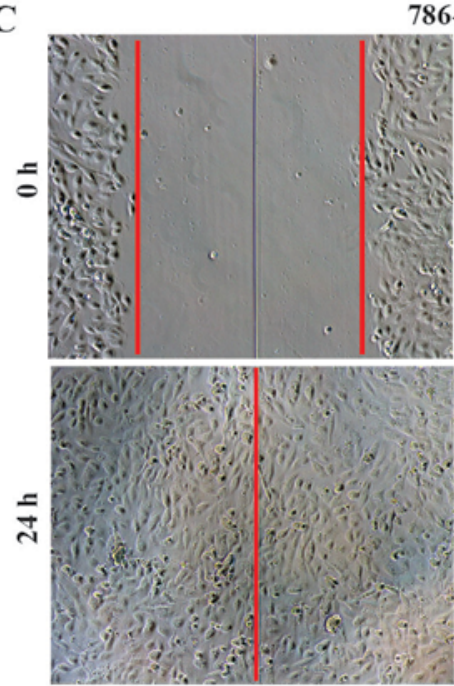

Control

E

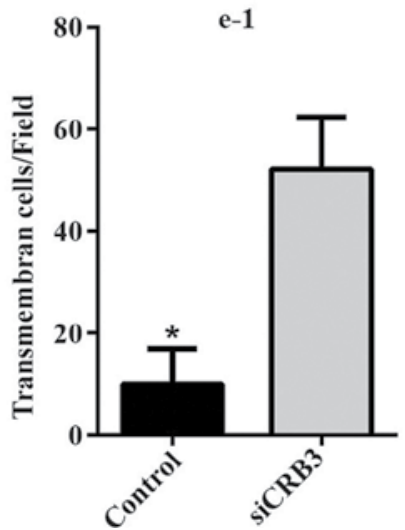

86-O
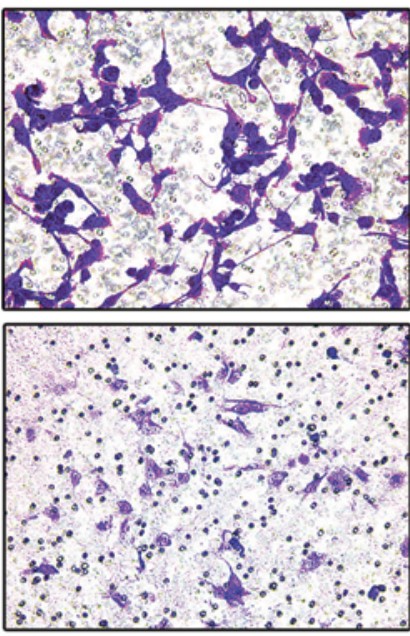

GV168-CRB3

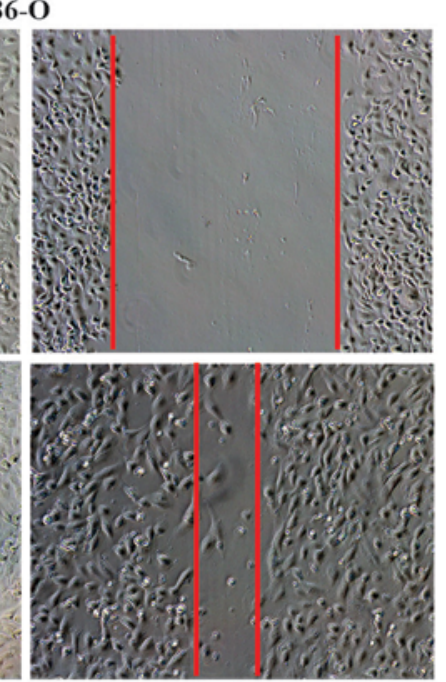

GV168-CRB3

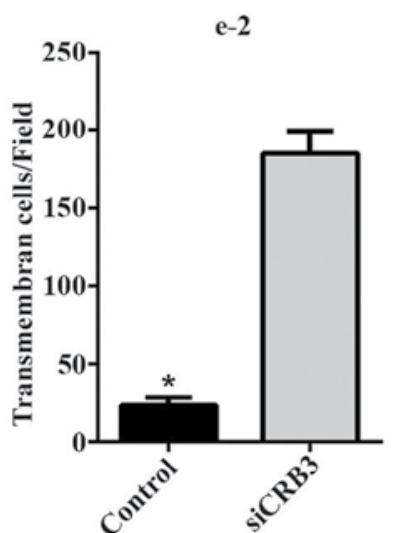

B
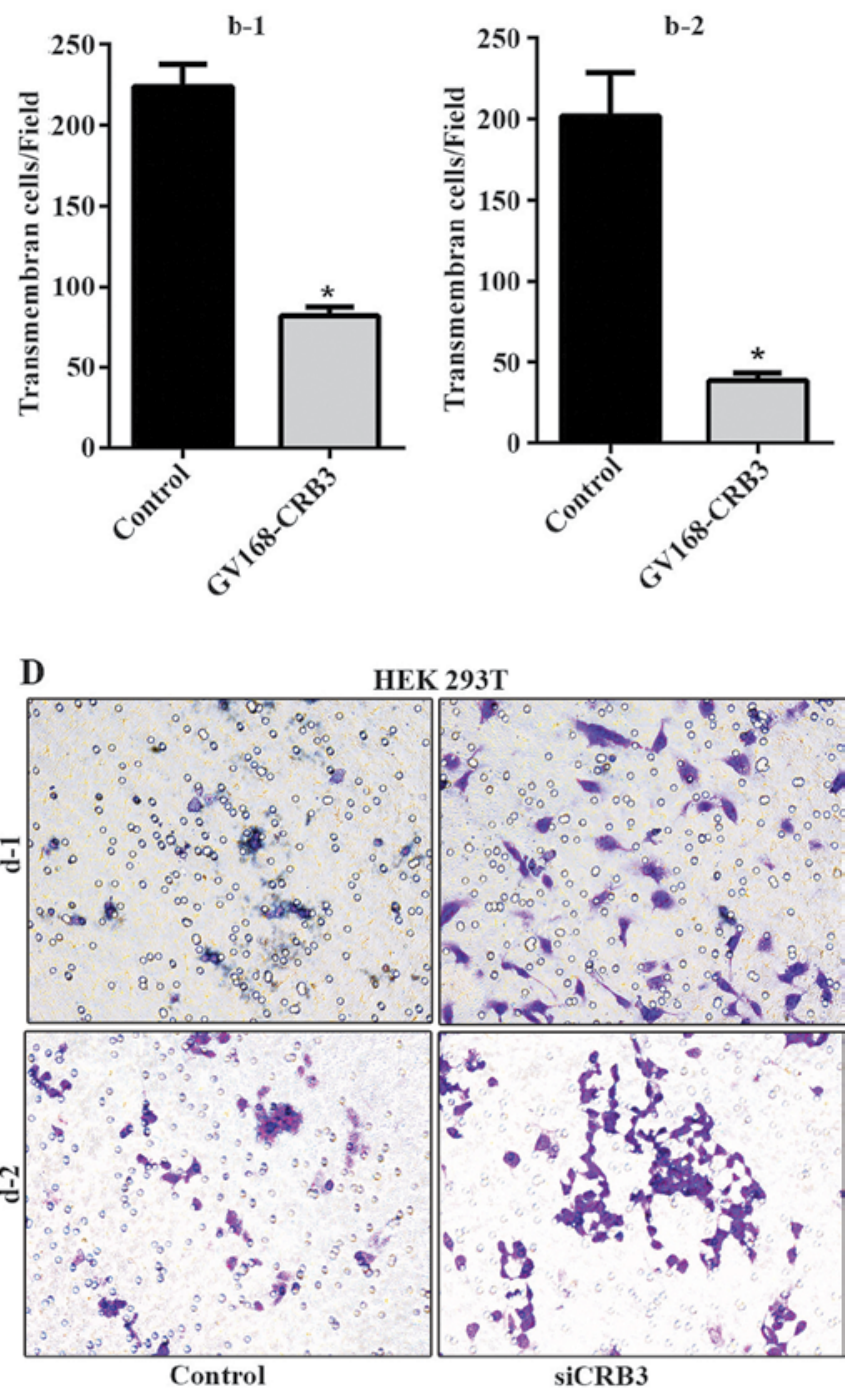

F

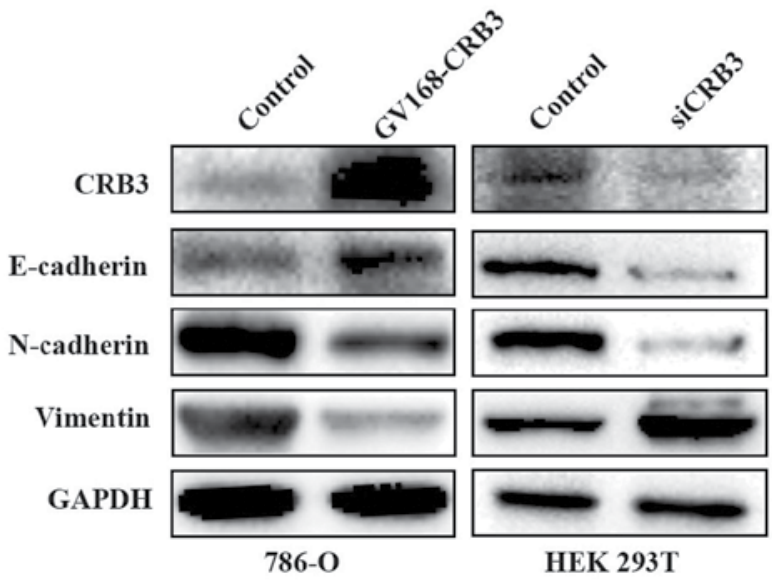

Figure 4. The Crumbs (CRB)3 in regulation of invasion and migration. (A and B) Migration and invasion of 786-O control cells and 786-O cells transfected with GV168-CRB3 plasmid were assessed by cell migration and invasion assays. (a-1 and b-1) Cell migration assay, (a-2 and b-2) cell invasion assay; each column in (B) represents the mean of the numbers of transmembrane cells in three randomly selected microscopic fields; The values were expressed as mean \pm SEM. ${ }^{*} \mathrm{P}<0.05$. (C) Migration of 786-O control cells and transfected 786-O cells were assessed by wound-healing assay. (D and E) Migration and invasion of human embryonic kidney 293T (HEK 293T) control cells and HEK 293T cells transfected with siRNA were assessed by cell migration and invasion assays. (d-1 and e-1) Cell migration assay, (d-2 and e-2) cell invasion assay; each column in (E) represents the mean of the numbers of transmembrane cells in three randomly selected microscopic fields; The values were expressed as mean \pm SEM. ${ }^{*} \mathrm{P}<0.05$. (F) Expression levels of CRB3 and epithelial-mesenchymal transition (EMT) markers in 786-O and HEK 293 T cells were assessed by western blot analysis.

junction formation and may contribute to invasion and metastasis, the hallmarks of malignant tumor progression and the main cause of deaths in cancer (33). Patients with negative CRB3 expression had a significantly shorter OS than patients 
with positive expressions. Therefore, loss of CRB3 and EMT may contribute to invasion and metastasis and cause the poor prognosis of the ccRCC patients. Interestingly, loss of CRB3 induced inconsistent changes of $\mathrm{N}$-cadherin expression in 786-O and HEK 293T cells. The cause of this disparity was not clear. Further studies are needed to solve this interesting problem.

Recent studies have also found that CRB3 is involved in tight junction formation in mammalian epithelial cells and that loss of CRB3 expression leads to tight junction defects $(30,40,41)$. Acquisition of tumorigenesis due to loss of CRB3 is associated with deficient tight junction formation and restoration of $\mathrm{CRB} 3$ expression restores junctions and polarity while suppressing migration and metastasis (22). Our results showed that overexpression of CRB3 restrained invasion and migration of 786-O cells. E-cadherin, essential for appropriate tight junction formation (42), was also increased in 786-O cells with CRB3 overexpression. These observations and data may suggest that CRB3 overexpression inhibited migration and invasion probably due to the proper tight junction formation. Further studies are needed to reveal the precise mechanism underlying the role of the increased CRB3 expression in inhibition of tumor migration and invasion.

Taken together, this study suggested that expression of CRB3 may be used as an independent favorable prognostic factor for patients with ccRCC. The identification of CRB3 as a prognostic factor may contribute to selection of high-risk patients for therapy, making it possible to improve the clinical outcome of the patients. It will be of great interest to investigate the roles of CRB3 expression in other human tumors and the precise molecular mechanisms underlying the functions of CRB3.

\section{Acknowledgements}

This study was financially supported by Grants from the National Natural Science Foundation of China (no. 81272342; Beijing, China) and Program for New Century Excellent Talents in University of the Ministry of Education of China (no. NCET-10-0677; Beijing, China) to Dr Peijun Liu.

\section{References}

1. Nelson WJ: Adaptation of core mechanisms to generate cell polarity. Nature 422: 766-774, 2003.

2. Tepass U, Theres $\mathrm{C}$ and Knust E: Crumbs encodes an EGF-like protein expressed on apical membranes of Drosophila epithelial cells and required for organization of epithelia. Cell 61: 787-799, 1990.

3. Tepass U and Knust E: Crumbs and stardust act in a genetic pathway that controls the organization of epithelia in Drosophila melanogaster. Dev Biol 159: 311-326, 1993.

4. Bhat MA, Izaddoost S, Lu Y, Cho KO, Choi KW and Bellen HJ: Discs Lost, a novel multi-PDZ domain protein, establishes and maintains epithelial polarity. Cell 96: 833-845, 1999.

5. Margolis B and Borg JP: Apicobasal polarity complexes. J Cell Sci 118: 5157-5159, 2005.

6. Suzuki A and Ohno S: The PAR-aPKC system: lessons in polarity. J Cell Sci 119: 979-987, 2006.

7. Bilder D: Epithelial polarity and proliferation control: links from the Drosophila neoplastic tumor suppressors. Genes Dev 18: 1909-1925, 2004.

8. Assémat E, Bazellières E, Pallesi-Pocachard E, Le Bivic A and Massey-Harroche D: Polarity complex proteins. Biochim Biophys Acta 1778: 614-630, 2008.
9. Nolan ME, Aranda V, Lee S, et al: The polarity protein Par6 induces cell proliferation and is overexpressed in breast cancer. Cancer Res 68: 8201-8209, 2008.

10. Eder AM, Sui X, Rosen DG, et al: Atypical PKCiota contributes to poor prognosis through loss of apical-basal polarity and cyclin E overexpression in ovarian cancer. Proc Natl Acad Sci USA 102: 12519-12524, 2005.

11. Regala RP, Weems C, Jamieson L, et al: Atypical protein kinase C iota is an oncogene in human non-small cell lung cancer. Cancer Res 65: 8905-8911, 2005.

12. Xue B, Krishnamurthy K, Allred DC and Muthuswamy SK: Loss of Par3 promotes breast cancer metastasis by compromising cell-cell cohesion. Nat Cell Biol 15: 189-200, 2013.

13. McCaffrey LM, Montalbano J, Mihai C and Macara IG: Loss of the Par3 polarity protein promotes breast tumorigenesis and metastasis. Cancer cell 22: 601-614, 2012.

14. Ellenbroek SI, Iden S and Collard JG: Cell polarity proteins and cancer. Semin Cancer Biol 22: 208-215, 2012.

15. Spaderna S, Schmalhofer O, Wahlbuhl M, et al: The transcriptional repressor ZEB1 promotes metastasis and loss of cell polarity in cancer. Cancer Res 68: 537-544, 2008.

16. Sandoval GJ, Graham DB, Gmyrek GB, et al: Novel mechanism of tumor suppression by polarity gene discs large 1 (DLG1) revealed in a murine model of pediatric B-ALL. Cancer Immunol Res 1: 426-437, 2013.

17. Etienne-Manneville S: Polarity proteins in migration and invasion. Oncogene 27: 6970-6980, 2008.

18. Dow LE and Humbert PO: Polarity regulators and the control of epithelial architecture, cell migration, and tumorigenesis. Int Rev Cytol 262: 253-302, 2007.

19. Martin-Belmonte F and Perez-Moreno M: Epithelial cell polarity, stem cells and cancer. Nat Rev Cancer 12: 23-38, 2011.

20. Dow LE, Elsum IA, King CL, Kinross KM, Richardson HE and Humbert PO: Loss of human Scribble cooperates with H-Ras to promote cell invasion through deregulation of MAPK signalling. Oncogene 27: 5988-6001, 2008.

21. Ling C, Zheng Y, Yin F, et al: The apical transmembrane protein Crumbs functions as a tumor suppressor that regulates Hippo signaling by binding to Expanded. Proc Natl Acad Sci USA 107: 10532-10537, 2010

22. Karp CM, Tan TT, Mathew R, et al: Role of the polarity determinant crumbs in suppressing mammalian epithelial tumor progression. Cancer Res 68: 4105-4115, 2008.

23. Athar U and Gentile TC: Treatment options for metastatic renal cell carcinoma: a review. Can J Urol 15: 3954-3966, 2008.

24. Cohen HT and McGovern FJ: Renal-cell carcinoma. N Engl J Med 353: 2477-2490, 2005.

25. Kaelin WG Jr: Treatment of kidney cancer: insights provided by the VHL tumor-suppressor protein. Cancer 115 (Suppl 10): S2262-S2272, 2009.

26. Kinouchi T, Kotake T, Mori Y and Abe T: Human renal cell carcinoma: establishment and characterization of a new cell line (OS-RC-2). In Vitro Cell Dev Biol 21: 195-199, 1985.

27. Fagerberg L, Hallström BM, Oksvold P, et al: Analysis of the human tissue-specific expression by genome-wide integration of transcriptomics and antibody-based proteomics. Mol Cell Proteomics 13: 397-406, 2014.

28. Bulgakova NA and Knust E: The Crumbs complex: from epithelial-cell polarity to retinal degeneration. J Cell Sci 122: 2587-2596, 2009

29. Makarova O, Roh MH, Liu CJ, Laurinec S and Margolis B: Mammalian Crumbs 3 is a small transmembrane protein linked to protein associated with Lin-7 (Pals1). Gene 302: 21-29, 2003.

30. Lemmers C, Michel D, Lane-Guermonprez L, et al: CRB3 binds directly to Par6 and regulates the morphogenesis of the tight junctions in mammalian epithelial cells. Mol Biol Cell 15: 1324-1333, 2004

31. Lu H and Bilder D: Endocytic control of epithelial polarity and proliferation in Drosophila. Nat Cell Biol 7: 1232-1239, 2005.

32. Roeth JF, Sawyer JK, Wilner DA and Peifer M: Rab11 helps maintain apical crumbs and adherens junctions in the Drosophila embryonic ectoderm. PLoS One 4: e7634, 2009.

33. Thiery JP and Sleeman JP: Complex networks orchestrate epithelial-mesenchymal transitions. Nat Rev Mol Cell Biol 7: 131-142, 2006.

34. Aigner K, Dampier B, Descovich L, et al: The transcription factor ZEB1 (deltaEF1) promotes tumour cell dedifferentiation by repressing master regulators of epithelial polarity. Oncogene 26: 6979-6988, 2007. 
35. Whiteman EL, Liu CJ, Fearon ER and Margolis B: The transcription factor snail represses Crumbs 3 expression and disrupts apico-basal polarity complexes. Oncogene 27: 3875-3879, 2008.

36. Gilles C, Polette M, Mestdagt M, et al: Transactivation of vimentin by beta-catenin in human breast cancer cells. Cancer Res 63: 2658-2664, 2003.

37. Onder TT, Gupta PB, Mani SA, Yang J,LanderES and Weinberg RA: Loss of E-cadherin promotes metastasis via multiple downstream transcriptional pathways. Cancer Res 68: 3645-3654, 2008.

38. Stoops SL, Pearson AS, Weaver C, et al: Identification and optimization of small molecules that restore E-cadherin expression and reduce invasion in colorectal carcinoma cells. ACS Chem Biol 6: 452-465, 2011
39. Weng W, Yin J,Zhang Y, Qiu J and Wang X: Metastasis-associated protein 1 promotes tumor invasion by downregulation of E-cadherin. Int J Oncol 44: 812-818, 2014

40. Fogg VC, Liu CJ and Margolis B: Multiple regions of Crumbs3 are required for tight junction formation in MCF10A cells. J Cell Sci 118: 2859-2869, 2005.

41. Roh MH, Fan S, Liu CJ and Margolis B: The Crumbs3-Pals1 complex participates in the establishment of polarity in mammalian epithelial cells. J Cell Sci 116: 2895-2906, 2003.

42. Tunggal JA, Helfrich I, Schmitz A, et al: E-cadherin is essential for in vivo epidermal barrier function by regulating tight junctions. EMBO J 24: 1146-1156, 2005. 